\title{
Random Fibonacci Sequences
}

\author{
Clément Sire ${ }^{1}$ and Paul L. Krapivsky ${ }^{2}$ \\ ${ }^{1}$ Laboratoire de Physique Quantique (UMR 5626 du CNRS), Université Paul Sabatier, 31062 Toulouse Cedex, France \\ ${ }^{2}$ Center for Polymer Physics and Department of Physics, Boston University, Boston MA 02215, USA
}

Solutions to the random Fibonacci recurrence $x_{n+1}=x_{n} \pm \beta x_{n-1}$ decrease (increase) exponentially, $x_{n} \sim \exp (\lambda n)$, for sufficiently small (large) $\beta$. In the limits $\beta \rightarrow 0$ and $\beta \rightarrow \infty$, we expand the Lyapunov exponent $\lambda(\beta)$ in powers of $\beta$ and $\beta^{-1}$, respectively. For the classical case of $\beta=1$ we obtain exact non-perturbative results. In particular, an invariant measure associated with Ricatti variable $r_{n}=x_{n+1} / x_{n}$ is shown to exhibit plateaux around all rational $r$ 's.

PACS numbers: 02.50.-r, 31.15.Md, 72.15.Rn

\section{INTRODUCTION}

The Fibonacci numbers $1,1,2,3,5,8,13, \ldots$ defined via $F_{n+1}=F_{n}+F_{n-1}$ abound in nature [1,2]. For example, they govern the number of leaves, petals and seed grains in plants [3]; they also give the number of ancestors of a drone. The Fibonacci recurrence is the simplest recurrence in which each number depends on the previous two and this perhaps explains why the Fibonacci sequence is so ubiquitous. A natural stochastic modification of the Fibonacci sequence is to allow both additions and substractions. Random Fibonacci sequences are related to many fields including condensed matter physics, dynamical systems, products of random matrices [4], continued fractions, etc. Random recurrences also form a chapter of the larger subject of iterated random functions [5].

The random Fibonacci recurrence $x_{n+1}=x_{n} \pm x_{n-1}$ results in sequences which behave erratically for small $n$. In the limit $n \rightarrow \infty$, however, exponential growth occurs with probability one as was established by Furstenberg [6] in 1963. The large $n$ behavior is characterized by the Lyapunov exponent $\lambda$,

$$
\lambda=\lim _{n \rightarrow \infty} \frac{\ln \left|x_{n}\right|}{n} .
$$

Exponential growth (decay) means that $\lambda$ is positive (negative). For the random Fibonacci recurrence where each \pm sign is independent and either + or - with probability $1 / 2$, the Lyapunov exponent is $\lambda=0.12397559 \ldots$ [7]. Exponential growth may seem unsurprising but similar generalized random Fibonacci recurrence,

$$
x_{n+1}=x_{n} \pm \beta x_{n-1},
$$

gives exponential growth only when the parameter $\beta$ is sufficiently large, $\beta>\beta_{s} \approx 0.70258$, whereas for $0<\beta<\beta_{s}$ solutions decay exponentially [8]. The decay occurs even though the expected values $\left\langle x_{n}\right\rangle$ remain constant and the expected values of the higher integer moments $\left\langle x_{n}^{2}\right\rangle,\left\langle x_{n}^{3}\right\rangle$, etc. grow exponentially. Indeed, from Eq. (2) one can deduce $\left\langle x_{n+1}^{2}\right\rangle=\left\langle x_{n}^{2}\right\rangle+\beta^{2}\left\langle x_{n-1}^{2}\right\rangle$ which implies $\left\langle x_{n}^{2}\right\rangle \sim\left[\frac{1}{2}+\left(\frac{1}{4}+\beta^{2}\right)^{1 / 2}\right]^{n}$, and similarly for higher moments [9]. Additionally, for $\beta>1 / 4$ an interesting non-smooth dependence of the Lyapunov exponent on the parameter $\beta$ has been observed [8] suggesting that the curve $\lambda(\beta)$ is a fractal (it remains unclear whether this curve becomes genuinely smooth for sufficiently large $\beta$ ). Similar non-smooth dependence on parameters has been reported for several disordered systems [10,11. Also, numerical results [8] suggest the following asymptotic behaviors of the Lyapunov exponent:

$$
\lambda \rightarrow \begin{cases}-\frac{1}{2} \beta^{2}-\frac{15}{8} \beta^{4} & \text { when } \beta \rightarrow 0, \\ \frac{1}{2} \ln \beta+\frac{0.114}{\beta} & \text { when } \beta \rightarrow \infty .\end{cases}
$$

While understanding of the nature of the curve $\lambda(\beta)$ might be a very difficult problem, one should be able to carry out asymptotic expansions of the Lyapunov exponent using tools developed in studies of one-dimensional disordered systems 10 17]. Indeed, Eq. (2) admits the standard interpretation in terms of the one-dimensional (discretized) Schrödinger equation. A peculiarity of the present problem is that the corresponding random Hamiltonian is non-Hermitian. Non-Hermitian random Hamiltonians appear in various non-equilibrium problems 18] and exhibit interesting behaviors 11922 , e.g., a delocalization transition may occur even in one dimension. Fortunately, tools developed for Hermitian problems can often be extended to the non-Hermitian case.

In the next Sect. II, we employ perturbation theory to expand the Lyapunov exponent in powers of $\beta$. In particular, we show that in the $\beta \rightarrow 0$ limit the second term is $-1.75 \times \beta^{4}$ rather than $-1.875 \times \beta^{4}$ [cf. Eq. (3)] and compute many more terms. In Sec. III, we use nonperturbative techniques to analyze the special $\beta=1$ case. In Sec. IV, we examine random sequences with Gaussian, rather than binary, disorder. Finally, a few open questions are discussed in the last Sec. V.

\section{PERTURBATION THEORY}

An old way of studying linear random recursions is to reduce them to random maps by introducing the Ricatti variable $R_{n}=x_{n+1} / x_{n}$. In the present case, Eq. (2) becomes

$$
R_{n}=1 \pm \frac{\beta}{R_{n-1}}
$$


The Lyapunov exponent is given by

$$
\lambda=\lim _{n \rightarrow \infty} \frac{1}{n} \sum_{j=1}^{n} \ln R_{j} .
$$

In the large $n$ limit, the probability distribution of $R_{n}$ becomes independent on $n$. The invariant distribution $P(R, \beta)$ satisfies

$$
P(R)=\frac{\beta}{2(R-1)^{2}}\left[P\left(\frac{\beta}{R-1}\right)+P\left(\frac{\beta}{1-R}\right)\right]
$$

which immediately follows from the random map (4). The analysis of the functional equation (6) often simplifies after transforming it into an integral equation

$$
\begin{aligned}
P(R, \beta)= & \int d R^{\prime} P\left(R^{\prime}, \beta\right) \\
& \times \frac{1}{2}\left[\delta\left(R-1+\frac{\beta}{R^{\prime}}\right)+\delta\left(R-1-\frac{\beta}{R^{\prime}}\right)\right] .
\end{aligned}
$$

Once we know the invariant distribution $P(R, \beta)$, we can employ Eq. (5) to compute the Lyapunov exponent

$$
\lambda(\beta)=\int d R P(R, \beta) \ln R .
$$

Depending on the magnitude of $\beta$, the support of the invariant distribution $P(R, \beta)$ may be either finite or infinite. Let us assume the former. Then the extreme values satisfy $R_{\min }=1-\beta / R_{\min }$ and $R_{\max }=1+\beta / R_{\min }$, or

$$
R_{\min }=\frac{1+\sqrt{1-4 \beta}}{2}, \quad R_{\max }=\frac{3-\sqrt{1-4 \beta}}{2} .
$$

Thus, the support is finite when the strength of disorder satisfies $\beta<1 / 4$. Furthermore, in this case the support is not merely the interval $\left[R_{\min }, R_{\max }\right]$ but rather a fractal set similar to the Cantor set. Indeed, the map $R \rightarrow 1 \pm \beta / R$ transforms the interval $\left[R_{\min }, R_{\max }\right]$ into the union of two subintervals, $\left[R_{\min }, 1-\beta / R_{\max }\right]$ and $\left[1+\beta / R_{\max }, R_{\max }\right]$. Restricting the map to these two subintervals shows that they are transformed into four subintervals. Proceeding in the same manner ad infinitum we construct the fractal support of the invariant distribution. On the other hand, the support is unbounded when $\beta>1 / 4$. This suggests to employ different perturbation approaches for small and large $\beta$.

\section{A. Weak disorder expansion}

When $\beta<1 / 4$, it is desirable to compute $\lambda(\beta)$ without explicit determination of the invariant distribution $P(R, \beta)$ which is a complicated singular function. The trick is to transform the integral in the basic relation (8) into an integral which can be calculated perturbatively using only the normalization requirement $\int d R P(R)=1$. To this end we insert Eq. (7) into Eq. (8) to yield

$$
\lambda=\frac{1}{2} \int d R P(R)\left[\ln \left(1-\frac{\beta}{R}\right)+\ln \left(1+\frac{\beta}{R}\right)\right] .
$$

Expanding the logarithms on the right-hand side of Eq. (10) we obtain

$$
\lambda=-\frac{\beta^{2}}{2} \int d R R^{-2} P(R)-\frac{\beta^{4}}{4} \int d R R^{-4} P(R)+\ldots
$$

In the limit $\beta \rightarrow 0$, the interval $\left[R_{\min }, R_{\max }\right]$ shrinks to $R=1$. Hence, the first integral on the right-hand side of the above equation approaches to $\int d R P(R)=1$. Thus $\lambda=-\beta^{2} / 2+\mathcal{O}\left(\beta^{4}\right)$ and the first term of the expansion was indeed derived without using an explicit form of the invariant distribution. However, the derivation of the next term is still impossible without knowledge of the invariant distribution. To avoid this we transform Eq. (10) as we transformed Eq. (8) into Eq. (10). Namely, we plug Eq. (7) into Eq. (10) to give

$$
\lambda=\frac{1}{4} \int d R P(R, \beta) L_{2}(R, \beta)
$$

where

$$
\begin{aligned}
L_{2}(R, \beta)= & \ln \left(1-\frac{\beta}{1-\frac{\beta}{R}}\right)+\ln \left(1-\frac{\beta}{1+\frac{\beta}{R}}\right) \\
& +\ln \left(1+\frac{\beta}{1+\frac{\beta}{R}}\right)+\ln \left(1+\frac{\beta}{1-\frac{\beta}{R}}\right) .
\end{aligned}
$$

Expanding the logarithms and inserting this expansion into Eq. (11) we obtain

$$
\lambda=-\frac{\beta^{2}}{2} \int d R P(R)-\frac{\beta^{4}}{4} \int d R\left(\frac{6}{R^{2}}+1\right) P(R)+\ldots
$$

The first integral is now identically equal to one, while the second approaches to 7 in the small $\beta$ limit. Therefore, $\lambda=-\beta^{2} / 2-7 \beta^{4} / 4+\mathcal{O}\left(\beta^{6}\right)$ is the two-term expansion. This shows that the small $\beta$ expansion of Eq. (3) is slightly incorrect.

Repeating the above procedure $k$ times, we recast Eq. (8) into

$$
\lambda=2^{-k} \int d R P(R, \beta) L_{k}(R, \beta),
$$

where

$$
L_{k}(R, \beta)=\sum_{\epsilon_{1}, \ldots, \epsilon_{k}} \ln \left[1 ; \epsilon_{1} \beta, 1, \epsilon_{2} \beta, \ldots, \epsilon_{k} \beta, R\right] .
$$

The sum on the right-hand side of Eq. (13) is taken over all $\epsilon_{j}= \pm 1$, and $[1 ; a, b, \ldots]$ denotes the continued fraction $1+\frac{a}{b+\ldots}$. Expanding $L_{k}(R, \beta)$ and plugging it into Eq. (12) one finds the correct expansion of the Lyapunov exponent up to $\mathcal{O}\left(\beta^{2 k}\right)$. A few of these terms can be 
computed by hand, and a bit more can be done with the help of Mathematica. One gets

$$
\begin{aligned}
\lambda(\beta)= & -\frac{1}{2} \beta^{2}-\frac{7}{4} \beta^{4}-\frac{29}{3} \beta^{6}-\frac{555}{8} \beta^{8} \\
& -\frac{2843}{5} \beta^{10}-\frac{30755}{6} \beta^{12}+\mathcal{O}\left(\beta^{14}\right) .
\end{aligned}
$$

The radius of convergence of this series appears to be equal to $1 / 4$ as one could guess from Eqs. (9). Hence the Lyapunov exponent is an analytic function of $\beta$ when $|\beta|<1 / 4$. Amusingly, the invariant distribution is a very singular function in this range of the parameter $\beta$. The series (14) perfectly reproduces numerical results [8] (5 representative digits), except for the case $\beta=1 / 4$ for which $\lambda_{\text {num }} \approx-0.0429$ and $\lambda_{\text {theor }} \approx-0.0424$. This slight discrepancy is due to the fact that for $\beta=1 / 4$ the generic term of the series decays algebraically in contrast with an exponential decay for $\beta<1 / 4$.

\section{B. Strong disorder expansion}

In the large $\beta$ limit, the support of the invariant distribution $P(R, \beta)$ is the whole real line. The trick which has been employed in the case of weak disorder does not apply, i.e., we cannot determine $\lambda(\beta)$ without knowledge of the invariant distribution. It proves convenient to use the modified Ricatti variable $r_{n}=\left|x_{n+1} / x_{n} \sqrt{\beta}\right|$. Then, Eq. (2) reduces to the random map

$$
r_{n}=\left|\frac{1}{r_{n-1}} \pm \delta\right|, \quad \delta \equiv \beta^{-1 / 2} .
$$

Once we know the invariant distribution $P(r, \delta)$, we can compute the Lyapunov exponent via Eq. (8) which now becomes

$$
\lambda=\frac{1}{2} \ln \beta+\int d r P(r) \ln r .
$$

The support of the invariant distribution is $0 \leq r<\infty$. It proves convenient to define $P(r)$ for negative $r$ via $P(r)=P(-r)$, so the support is the entire real line. In present notations, the functional equation (6) becomes

$2 P(r)=\frac{1}{(r+\delta)^{2}} P\left(\frac{1}{r+\delta}\right)+\frac{1}{(r-\delta)^{2}} P\left(\frac{1}{r-\delta}\right)$.

For $\delta=0$, this equation reduces to $P(r)=r^{-2} P(1 / r)$ which has infinitely many solutions. For $\delta>0$, however, the invariant distribution is unique. Thus taking the $\delta \rightarrow 0$ limit of the invariant distribution $P(r, \delta)$ we shall obtain a unique appropriate solution. In this sense, we are building a degenerate perturbation approach.

To determine $P(r, \delta)$, notice that Eq. (17) can be rewritten as

$$
P(r)=r^{-2} P(1 / r)+\frac{1}{2} D_{\delta}^{2}\left[r^{-2} P(1 / r)\right],
$$

where $D_{\delta}^{2}$ is the discrete analog of the second derivative, $D_{\delta}^{2} F(r)=F(r+\delta)-2 F(r)+F(r-\delta)$. Expanding the right-hand side of Eq. (18) into Taylor series and denoting $D=\frac{d}{d r}$ we obtain

$$
P(r)=\sum_{k=0}^{\infty} \frac{\delta^{2 k}}{(2 k) !} D^{2 k}\left[r^{-2} P(1 / r)\right] .
$$

The change of variable $r \rightarrow 1 / r$ transforms Eq. (19) into

$$
P(1 / r)=\sum_{k=0}^{\infty} \frac{\delta^{2 k}}{(2 k) !} \mathcal{D}^{2 k}\left[r^{2} P(r)\right]
$$

where $\mathcal{D}=r^{2} \frac{d}{d r}$. If we now plug Eq. (20) into Eq. (19), we eliminate $P(1 / r)$ and thus arrive at a closed equation for the invariant distribution $P(r, \delta)$ :

$$
P(r)=\sum_{k=0}^{\infty} \sum_{l=0}^{\infty} \frac{\delta^{2 k+2 l}}{(2 k) !(2 l) !} D^{2 k}\left\{r^{-2} \mathcal{D}^{2 l}\left[r^{2} P(r)\right]\right\} .
$$

Equation (21) suggests to seek a solution $P(r, \delta)$ which can be expanded as a power series in $\delta^{2}$ :

$$
P(r, \delta)=\sum_{j=0}^{\infty} \delta^{2 j} P_{j}(r) .
$$

Plugging the series (22) into Eq. (21) one finds that the terms of order one cancel. Equating terms of order $\delta^{2}$ leads to the following second order differential equation for $P_{0}(r)$

$$
D \mathcal{L}_{1} P_{0}(r)=0 \quad \text { with } \quad \mathcal{L}_{1} \equiv D+r^{2} D r^{2} .
$$

Integrating once we arrive at $\mathcal{L}_{1} P_{0}=0$, the integration constant being equal to zero to ensure that $P_{0}(r)$ vanishes in the large $r$ limit. This can be re-written as

$$
\left(1+r^{4}\right) P_{0}^{\prime}(r)+2 r^{3} P_{0}(r)=0,
$$

whose normalized solution is

$$
P_{0}(r)=\frac{2 \sqrt{\pi}}{\Gamma^{2}(1 / 4)} \frac{1}{\sqrt{1+r^{4}}} .
$$

Thus among infinitely many invariant distributions satisfying the duality relation $P(r)=r^{-2} P(1 / r)$ we have indeed selected the specific solution (25). Note that $\int d r P_{0}(r) \ln r=0$ (this is actually valid for any function which obeys the duality relation). Therefore, the anticipated contribution of order one to the Lyapunov exponent [cf. Eq. (16)] is actually equal to zero.

Similarly equating the terms of order $\delta^{4}$ one finds $D \mathcal{L}_{1} P_{1}+D \mathcal{L}_{3} P_{0}=0$, where $\mathcal{L}_{3}$ is certain third order differential operator. Integrating the above equation yields $\mathcal{L}_{1} P_{1}+\mathcal{L}_{3} P_{0}=0$ (the integration constant is equal to zero to ensure that $P_{1}(r)$ vanishes in the large $r$ limit). 
Recalling that $\mathcal{L}_{1} P_{0}=0$ one can simplify the term $\mathcal{L}_{3} P_{0}$. The final first order differential equation for $P_{1}(r)$ reads

$$
\mathcal{L}_{1} P_{1}(r)=\frac{\left(5+r^{4}\right) D^{3}+2 r^{3} D^{2}}{12} P_{0}(r) .
$$

It is helpful to write $P_{1}(r)=P_{0}(r) f_{1}(r)$ and then exploit the identity $\mathcal{L}_{1}\left[P_{0}(r) f_{1}(r)\right] \equiv\left(1+r^{4}\right) P_{0}(r) f_{1}^{\prime}(r)$. Integrating the resulting equation yields

$$
f_{1}(r)=c_{1}+R_{1}(r), \quad R_{1}(r)=\frac{r^{2}\left(3 r^{8}+8 r^{4}-15\right)}{6\left(r^{4}+1\right)^{3}} .
$$

The integration constant $c_{1}$ can be found from the relation $\int d r P_{1}(r)=0$ which ensures that the normalization condition $\int d r P(r)=1$ holds. We get

$$
c_{1}=\left[\frac{\Gamma(3 / 4)}{\Gamma(1 / 4)}\right]^{2} .
$$

A direct computation gives $\int d r P_{1}(r) \ln r=c_{1}$. Thus, in this order the contribution to the Lyapunov exponent is equal to $c_{1} \beta^{-1}$.

To determine $P_{j}(r)$, we repeat the above procedure. Plugging (22) into (21), equating terms of order $\delta^{2 j+2}$, and integrating once the resulting equation yields

$$
\mathcal{L}_{1} P_{j}(r)+\sum_{n=1}^{j} \mathcal{L}_{2 n+1} P_{j-n}(r)=0
$$

where $\mathcal{L}_{2 n+1}$ is the differential operator of order $2 n+1$,

$$
\begin{aligned}
\mathcal{L}_{2 n+1} & =\frac{2}{(2 n+2) !} D^{2 n+1} \\
& +\sum_{k=0}^{n} \frac{2}{(2 k) !(2 n-2 k+2) !} D^{2 k} \mathcal{D}^{2(n-k)+1} r^{2} .
\end{aligned}
$$

Equations (29) can be solved recursively. Writing again $P_{j}(r)=f_{j}(r) P_{0}(r)$, we find in the second order

$$
\begin{aligned}
f_{2}(r) & =-\frac{1}{96}+c_{1} f_{1}(r)+R_{2}(r), \\
R_{2}(r) & =\frac{r^{4}\left(463-3640 r^{4}+2514 r^{8}+440 r^{12}-17 r^{16}\right)}{24\left(r^{4}+1\right)^{6}} .
\end{aligned}
$$

In the third order we have

$$
\begin{aligned}
f_{3}(r) & =\frac{14699}{21600} c_{1}-\frac{1}{96} f_{1}(r)+c_{1} f_{2}(r)+R_{3}(r) \\
R_{3}(r) & =\frac{r^{2} Q_{3}(r)}{15120\left(r^{4}+1\right)^{9}} \\
Q_{3}(r) & =11340 r^{32}-678825 r^{28}-11260368 r^{24} \\
& -3619377 r^{20}+356871272 r^{16}-471736467 r^{12} \\
& +125696592 r^{8}-5587155 r^{4}+11340 .
\end{aligned}
$$

The constants were determined recursively after a long computation exploiting the normalization requirements $\int d r P_{j}(r)=0$ and the basic identity $\Gamma(1+z)=z \Gamma(z)$ for the gamma function 23].

After inserting above expressions for $P_{j}(r)$ in Eq. (16), we arrive at the expansion

$$
\lambda(\beta)=\frac{1}{2} \ln \beta+\sum_{k=1}^{\infty} a_{k} \beta^{-k} .
$$

One can in principle generate $\lambda(\beta)$ to any order. The first few coefficients are

$$
\begin{aligned}
& a_{1}=c_{1}=0.1142366452611159 \ldots \\
& a_{2}=c_{1}^{2}+\frac{1}{144}=0.01999445564958 \ldots \\
& a_{3}=0.0105345239 \ldots \\
& a_{4}=0.0176632096 \ldots
\end{aligned}
$$

The coefficients $a_{i}$ 's have extremely complicated analytical expressions in terms of the $\Gamma$ function which we have not been able to simplify (although it appears plausible that these expressions can be reduced to polynomials of $c_{1}$ with rational coefficients). The exact value for $a_{1}$ is in good agreement with the numerical estimate from Ref. [8]. From the first four coefficients, one could guess that the radius of the convergence of series (30) is of order unity. However, an apparent fractal structure of the curve $\lambda(\beta)$ makes the above guess questionable. Even if our strong disorder expansion is asymptotic, it is quite accurate as can be seen by comparison of the four-term expansion (30) with numerical results. For $\beta=8$, both give $\lambda \approx 1.05433$ whereas there is a slight discrepancy for $\beta=4$ as $\lambda_{\text {num }} \approx 0.72309$ and $\lambda_{\text {theor }} \approx 0.72319$. On Fig. 1, we compare analytical (third-order expansion) and numerical results for the invariant distribution.

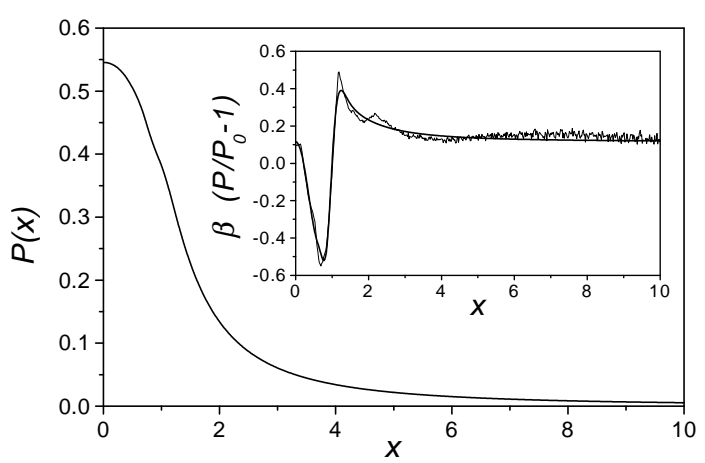

FIG. 1. $P_{\text {num }}$, which is obtained after $2 \times 10^{9}$ iterations of the map (15), and $P_{\text {theor }}$, which is the exact third order expansion in $\beta$, are plotted for $\beta=10$ (curves are indistinguishable). The inset compares $\beta\left(P_{\text {num }} / P_{0}-1\right)$ to $\beta\left(P_{\text {theor }} / P_{0}-1\right)$ (thick line). The Lyapunov exponent is $\lambda_{\text {num }} \approx \lambda_{\text {theor }} \approx 1.16293$. 


\section{THE GOLDEN MEAN CASE: $\beta=1$}

\section{A. Generalities}

We now focus on the particular case $\beta=1$ which admits a non-perturbative treatment. An ingenious construction of the invariant distribution $P(r)$ and the invariant measure $\nu([a, b])=\int_{a}^{b} d r P(r)$ which involves a Stern-Brocot division of the real line has been proposed by Viswanath [7]. In this subsection we first recall that construction [7] and the definition of the Stern-Brocot division (for details, see Ref. [24] and also Ref. [1] which describes closely related Farey series). We then derive useful symmetry relations which will lead to new quantitative results concerning the invariant distribution $P$ and the invariant measure $\nu$.

The invariant distribution $P(r)$ is symmetric, so we can limit ourselves to the half-line $r \geq 0$. The SternBrocot division is defined as follows: Start with the halfline $I_{0}=\left[\frac{0}{1}, \frac{1}{0}\right]$ and divide it into two intervals, $\left[\frac{0}{1}, \frac{1}{1}\right]$ and $\left[\frac{1}{1}, \frac{1}{0}\right]$; then divide every first generation interval and continue in this manner. Generally, the mediant of an interval $\left[p / q, p^{\prime} / q^{\prime}\right]$ is obtained by "Farey addition":

$$
\frac{p}{q} \oplus \frac{p^{\prime}}{q^{\prime}}=\frac{p+p^{\prime}}{q+q^{\prime}}
$$

Thus, the interval $\left[p / q, p^{\prime} / q^{\prime}\right]$ is divided according to the rule

$$
\left[\frac{p}{q}, \frac{p^{\prime}}{q^{\prime}}\right] \longrightarrow\left[\frac{p}{q}, \frac{p+p^{\prime}}{q+q^{\prime}}\right] \bigcup\left[\frac{p+p^{\prime}}{q+q^{\prime}}, \frac{p^{\prime}}{q^{\prime}}\right]
$$

This generates $2^{n}$ intervals at the $n^{\text {th }}$ generation, each interval $I$ of the $(n-1)^{\text {th }}$ generation producing a left and a right child $(\mathcal{L} I$ and $\mathcal{R} I)$ in the $n^{\text {th }}$ generation, according to Eq. (32). For instance, $\left[\frac{1}{3}, \frac{1}{2}\right]=\mathcal{R} \mathcal{L} \mathcal{L} I_{0}$, and generally every Stern-Brocot interval $I$ can be presented as $I=\mathcal{S} I_{0}$, where $\mathcal{S}$ is the unique sequence of $\mathcal{L}$ 's and $\mathcal{R}$ 's. The length of $\mathcal{S}$ is denoted $|\mathcal{S}|$. Using this representation one can prove numerous properties of the Stern-Brocot division, e.g., the assertion that for every Stern-Brocot interval $\left[\frac{p}{q}, \frac{p^{\prime}}{q^{\prime}}\right]$, the identity $p^{\prime} q-p q^{\prime}=1$ holds [24].

The invariance condition, i.e. Eq. (17) with $\delta=1$, can be re-written in terms of the invariant measure to give

$\nu(a, b)=\frac{1}{2}\left\{\nu\left(\frac{1}{-1+b}, \frac{1}{-1+a}\right)+\nu\left(\frac{1}{1+b}, \frac{1}{1+a}\right)\right\}$.

Making use of the above equation, it is possible to express the measure of the left and right children via the measure of the parent interval [7]:

$$
\nu\left(\mathcal{L S} I_{0}\right)= \begin{cases}(1-\tau) \nu\left(\mathcal{S} I_{0}\right) & \text { if }|\mathcal{S}| \text { is even } \\ \tau \nu\left(\mathcal{S} I_{0}\right) & \text { if }|\mathcal{S}| \text { is odd }\end{cases}
$$

and

$$
\nu\left(\mathcal{R S} I_{0}\right)= \begin{cases}\tau \nu\left(\mathcal{S} I_{0}\right) & \text { if }|\mathcal{S}| \text { is even } \\ (1-\tau) \nu\left(\mathcal{S} I_{0}\right) & \text { if }|\mathcal{S}| \text { is odd }\end{cases}
$$

where $\tau=(\sqrt{5}-1) / 2$ is the golden ratio (which is also the inverse growth constant of the deterministic Fibonacci numbers, $F_{n} \sim \tau^{-n}$ ).

We now turn to the derivation of symmetry relations which will be helpful later. To obtain the first one we take an arbitrary Stern-Brocot interval $\mathcal{S} I_{0}=\left[\frac{p}{q}, \frac{p^{\prime}}{q^{\prime}}\right]$ and notice that the Stern-Brocot interval $\mathcal{S} \mathcal{R} \mathcal{R} I_{0}=\left[\frac{p}{q}+2, \frac{p^{\prime}}{q^{\prime}}+2\right]$ differs from $\mathcal{S} I_{0}$ by a mere translation. Using Eqs. 33. (34) one finds $\tau(1-\tau) \nu\left(\mathcal{S} I_{0}\right)=\nu\left(\mathcal{S} \mathcal{R} \mathcal{R} I_{0}\right)$, i.e.,

$$
\nu\left(\left[\frac{p}{q}+2, \frac{p^{\prime}}{q^{\prime}}+2\right]\right)=\rho \nu\left(\left[\frac{p}{q}, \frac{p^{\prime}}{q^{\prime}}\right]\right),
$$

with $\rho=\tau(1-\tau)=\sqrt{5}-2$.

Note that every interval with rational end points can be formed by joining appropriate Stern-Brocot intervals. Hence Eq. (35) holds for any rational interval $\left[\frac{p}{q}, \frac{p^{\prime}}{q^{\prime}}\right]$. Since rational numbers form a dense set on the line and $\nu(r) \equiv \nu([0, r])$ is a continuous (increasing) function, Eq. (35) applies to arbitrary intervals. Specializing to the interval $[0, r]$, we arrive at the first relation

$$
\nu([2, r+2])=\rho \nu(r),
$$

which can be also presented as

$$
P(r+2)=\rho P(r) \quad \text { for } \quad r \geq 0 .
$$

The second symmetry relation,

$$
P(2-r)=P(r), \quad \text { for } \quad 0 \leq r \leq 2,
$$

expresses a mirror symmetry with respect to 1 in the interval $[0,2]$. To prove this result, we take a Stern-Brocot interval $\mathcal{S} \mathcal{L} I_{0}=\left[p / q, p^{\prime} / q^{\prime}\right] \subset[0,1]$ and notice that its symmetric image $\left[2-p^{\prime} / q^{\prime}, 2-p / q\right] \subset[1,2]$ is also a Stern-Brocot interval. Specifically, the symmetric interval can be presented as $\overline{\mathcal{S}} \mathcal{L} \mathcal{R} I_{0}$, where $\overline{\mathcal{S}}$ is the sequence obtained from $\mathcal{S}$ by exchanging the $\mathcal{L}$ 's and $\mathcal{R}$ 's. It turns out that the interval and its symmetric image have the same invariant measures

$$
\nu\left(\mathcal{S} \mathcal{L} I_{0}\right)=\nu\left(\overline{\mathcal{S}} \mathcal{L} \mathcal{R} I_{0}\right) .
$$

One then deduces Eq. (38) from Eq. (39) repeating the argument which has been employed in deducing Eq. 37) from Eq. (35).

The proof of Eq. (39) can be accomplished by induction on the length. Suppose that Eq. (39) holds for sequences $\mathcal{S}$ of length $n$. Assuming $n$ even and taking the right child of $\mathcal{S} \mathcal{L} I_{0}$, we use Eq. (34) to yield

$$
\begin{gathered}
\nu\left(\mathcal{R} \mathcal{S} \mathcal{L} I_{0}\right)=(1-\tau) \nu\left(\mathcal{S} \mathcal{L} I_{0}\right), \\
\nu\left(\mathcal{L} \overline{\mathcal{S}} \mathcal{L} \mathcal{R} I_{0}\right)=(1-\tau) \nu\left(\overline{\mathcal{S}} \mathcal{L} \mathcal{R} I_{0}\right) .
\end{gathered}
$$

Since $\mathcal{L}=\overline{\mathcal{R}}$ and right-hand sides of above equations are equal, we obtain $\nu\left(\mathcal{R} \mathcal{S} \mathcal{L} I_{0}\right)=\nu\left(\overline{\mathcal{R}} \overline{\mathcal{S}} \mathcal{L} \mathcal{R} I_{0}\right)$, thus proving induction step for even $n$ and the right child. The three other cases can be proved in a similar way. 


\section{B. Analytical results}

In this subsection, we will make use of the above symmetries to obtain quantitative results for the invariant distribution $P$ and the invariant measure $\nu$. For instance, one can readily establish extreme behaviors. From Eqs. (33)-(34), one finds

$$
\nu\left(\mathcal{L}^{2 n} I_{0}\right)=\nu\left(\mathcal{R}^{2 n} I_{0}\right)=\rho^{n} \nu\left(I_{0}\right)
$$

for any (positive) integer $n$. Clearly, $\mathcal{L}^{2 n} I_{0}=\left[0, \frac{1}{2 n}\right]$ and $\mathcal{R}^{2 n} I_{0}=[2 n, \infty]$. Additionally, $\nu\left(I_{0}\right)=1 / 2$ which immediately follows from normalization. Thus Eq. (42) reduces to

$$
\nu\left(\frac{1}{2 n}\right)=\nu([2 n, \infty])=\frac{1}{2} \rho^{n} .
$$

From Eq. (43) we deduce the small $r$ behavior,

$$
\nu(r) \sim e^{-c / r} \quad \text { with } \quad c=-\frac{1}{2} \ln \rho,
$$

and the large $r$ behavior,

$$
\frac{1}{2}-\nu(r) \sim e^{-c r} .
$$

Thus, the invariant measure $\nu(r)$ sharply vanishes when $r \rightarrow 0$. Remarkably, similar plateaux exist around all rational $r$ 's. It is useful to demonstrate the existence of the plateau at the proximity of a simple rational point, say $r=1 / 2$. Take the family of intervals $\mathcal{L}^{2 n} \mathcal{R} \mathcal{L} I_{0}=\left[\frac{1}{2}, \frac{2 n+1}{4 n+1}\right]$. The length of the $n^{\text {th }}$ interval is $\epsilon_{n}=[2(4 n+1)]^{-1}$. Exploiting Eqs. (33)-(34), we can compute the measure of these intervals and then examine how the measure scales with length. We get

$$
\nu\left(\left[\frac{1}{2}, \frac{2 n+1}{4 n+1}\right]\right)=\frac{(1-\tau)^{2}}{2} \rho^{n} \sim e^{-c / 4 \epsilon_{n}} .
$$

The continuity of the measure implies that the scaling holds for all small intervals, $\nu\left(\frac{1}{2}+\epsilon\right)-\nu\left(\frac{1}{2}\right) \sim e^{-c / 4 \epsilon}$.

Similarly, one can work out the proximity of an arbitrary rational number. One finds that the measure of the interval $[p / q, p / q+\epsilon]$ ( $p$ and $q$ are mutually prime integers) exhibits the following behavior

$$
\nu\left(\frac{p}{q}+\epsilon\right)-\nu\left(\frac{p}{q}\right) \sim \exp \left(-\frac{c}{q^{2} \epsilon}\right),
$$

with $c=-\frac{1}{2} \ln \rho$ as above. Similar asymptotics apply when we approach the rational point from the left.

Turn now to the invariant distribution $P(r)$. This function obeys a striking number of intricate identities. [Note that it is a slight abuse of language to speak of $P$ as a function: $P$ is a distribution rather than a function.] In the following, we will be using Eq. (17), with $\delta=1$, so we write it down for easy reference

$$
2 P(r+1)=\frac{1}{r^{2}} P\left(\frac{1}{r}\right)+\frac{1}{(r+2)^{2}} P\left(\frac{1}{r+2}\right) .
$$

Note that Eq. (48) at $r+2 n$ can be written as

$$
2 P(r+2 n+1)=U_{n}(r)+U_{n+1}(r),
$$

where we used the shorthand notation

$$
U_{n}(r)=\frac{1}{(r+2 n)^{2}} P\left(\frac{1}{r+2 n}\right) .
$$

Changing $n$ to $n+1$ in Eq. (49) gives

$$
2 P(r+2 n+3)=U_{n+1}(r)+U_{n+2}(r) .
$$

Recalling that $P(r+2 n+3)-\rho P(r+2 n+1)=0$ [this is Eq. (37) at $r+2 n]$, we reduce (49), (51) to a recursion which involves only $U$ 's:

$$
U_{n+2}(r)+(1-\rho) U_{n+1}(r)-\rho U_{n}(r)=0 .
$$

Since the variable $r$ is mute, Eq. (52) is merely a linear recursion which is straightforwardly solved to find two independent solutions, $(-1)^{n}$ and $\rho^{n}$. Therefore, the general solution is

$$
\frac{1}{(r+2 n)^{2}} P\left(\frac{1}{r+2 n}\right)=A(r)(-1)^{n}+B(r) \rho^{n} .
$$

In the $n \rightarrow \infty$ limit, the left-hand side of Eq. (53) approaches to zero. Thus, $A(r)=0$. Another coefficient $B(r)$ is found by specializing Eq. (53) to $n=0$. Equation (53) therefore reduces to

$$
\frac{1}{(r+2 n)^{2}} P\left(\frac{1}{r+2 n}\right)=\frac{\rho^{n}}{r^{2}} P\left(\frac{1}{r}\right) .
$$

One can derive a few more useful formulae relating $P(r)$ at different points. Performing the change of variable $r \rightarrow r^{-1}$, one transforms Eq. (54) into

$$
P(r)=\frac{\rho^{-n}}{(1+2 n r)^{2}} P\left(\frac{r}{1+2 n r}\right) .
$$

One can also take Eq. (54) at $n=1$ and insert it into Eq. (48). The outcome reads

$$
P(r+1)=\frac{1+\rho}{2 r^{2}} P\left(\frac{1}{r}\right)
$$

Take now Eq. (56), change the variable $r \rightarrow r+1$, and use Eq. (37). This transforms Eq. (56) into

$$
P(r)=\left(\frac{1+\tau}{1+r}\right)^{2} P\left(\frac{1}{1+r}\right) .
$$

We can take the same identity with $(1+r)^{-1}$ instead of $r$ and insert it into the right-hand side of Eq. (57) thus obtaining another identity. Proceeding in this manner, we arrive at a series of identities 


$$
P(r)=\frac{(1+\tau)^{2 m}}{\left(F_{m}+r F_{m-1}\right)^{2}} P\left(\frac{F_{m-1}+r F_{m-2}}{F_{m}+r F_{m-1}}\right) .
$$

These identities apply for all integer $m$ 's as well as the Fibonacci numbers $F_{m}$ which are defined for all integer $m$, e.g., $F_{-4}=2, F_{-3}=-1, F_{-2}=1, F_{-1}=0, F_{0}=1$.

Replacing $r \rightarrow 2-r$ in identities (58) and using the symmetry relation (38) one obtains another infinite series of identities. The simplest such identity (corresponding to $m=1$ ) reads

$$
P(r)=\left(\frac{1+\tau}{3-r}\right)^{2} P\left(\frac{1}{3-r}\right) .
$$

The above identities together with the basic relation $\nu([a, b])=\int_{a}^{b} d r P(r)$ can be used to re-derive all previous results about the invariant measure, including the most interesting findings about the plateaux.

\section{Generating the invariant measure}

Because of symmetries, it is sufficient to study $\nu(r)$ on the interval $[0,1]$. To distinguish the restricted version from the general case we change the notation: $r \rightarrow x$, $P \rightarrow \frac{1-\rho}{4} f, \nu \rightarrow \frac{1-\rho}{4} N$. The prefactor $(1-\rho) / 4$ guarantees that the invariant measure $N(x)=\int_{0}^{x} d y f(y)$ obeys $N(1)=1$. To see this, we take the normalization condition, $1=\int_{-\infty}^{\infty} d r P(r)$, and massage it a bit:

$$
2 \int_{0}^{\infty} d r P(r)=\frac{2}{1-\rho} \int_{0}^{2} d r P(r)=\frac{4}{1-\rho} \int_{0}^{1} d r P(r) .
$$

The first above identity is obtained by using Eq. (37) and summing up the geometric series while the last is an obvious consequence of Eq. (38). Thus we indeed obtain $N(1)=1$ if we choose $f(x)=\frac{4}{1-\rho} P(x)$.

Now let us integrate $f(x) F(x)$ with any $F(x)$ over intervals $(0,1 / 3),(1 / 3,1 / 2),(1 / 2,1)$. We find

$$
\begin{aligned}
\int_{0}^{1 / 3} d x f F & =\int_{0}^{1} \frac{d x}{(1+2 x)^{2}} f\left(\frac{x}{1+2 x}\right) F\left(\frac{x}{1+2 x}\right) \\
& =\rho \int_{0}^{1} d x f(x) F\left(\frac{x}{1+2 x}\right), \\
\int_{1 / 3}^{1 / 2} d x f F & =\int_{0}^{1} \frac{d x}{(3-x)^{2}} f\left(\frac{1}{3-x}\right) F\left(\frac{1}{3-x}\right) \\
& =(1+\tau)^{-2} \int_{0}^{1} d x f(x) F\left(\frac{1}{3-x}\right), \\
\int_{1 / 2}^{1} d x f F & =\int_{0}^{1} \frac{d x}{(1+x)^{2}} f\left(\frac{1}{1+x}\right) F\left(\frac{1}{1+x}\right) \\
& =(1+\tau)^{-2} \int_{0}^{1} d x f(x) F\left(\frac{1}{1+x}\right) .
\end{aligned}
$$

In deriving the first lines in above formulae we have used the mappings $x(1+2 x)^{-1},(3-x)^{-1},(1+x)^{-1}$ of the unit interval $(0,1)$ onto the intervals which appear on the lefthand side. We then exploited Eq. (55) at $n=1$, Eq. (59), and Eq. (57), respectively, to obtain the second lines.

Summing up Eqs. (60)-62) leads to identity

$$
\int_{0}^{1} d x f(x) F(x)=\int_{0}^{1} d x f(x) \hat{T}[F(x)]
$$

where the linear operator $\hat{T}$ acts on $F$ according to

$$
\begin{aligned}
& \hat{T}[F(x)]=\sum_{i=1}^{3} \alpha_{i} F\left(h_{i}(x)\right) \\
& \alpha_{1}=\rho, \quad \alpha_{2}=\alpha_{3}=(1+\tau)^{-2}, \quad \sum_{i=1}^{3} \alpha_{i}=1 \\
& h_{1}(x)=\frac{x}{1+2 x}, \quad h_{2}(x)=\frac{1}{3-x}, \quad h_{3}(x)=\frac{1}{1+x} .
\end{aligned}
$$

These relations show that the distribution $f$ can be generated by the following simple recursion process. We take an arbitrary point $x \in(0,1]$ and assign a unit weight $w(x)=1$. We then define three new points and associated weights according to the rule

$$
x_{i}=h_{i}(x), \quad w\left(x_{i}\right)=\alpha_{i} w(x), \quad i=1,2,3 .
$$

Iterating $n$ times, we get $3^{n}$ different $x_{i}$ 's. The weights $w\left(x_{i}\right)$ of these points add up to 1 and $w(x)$ converge (in the weak sense) to $f(x)$. In Fig. 2, we plot the invariant measure obtained after 4 and 10 iterations.

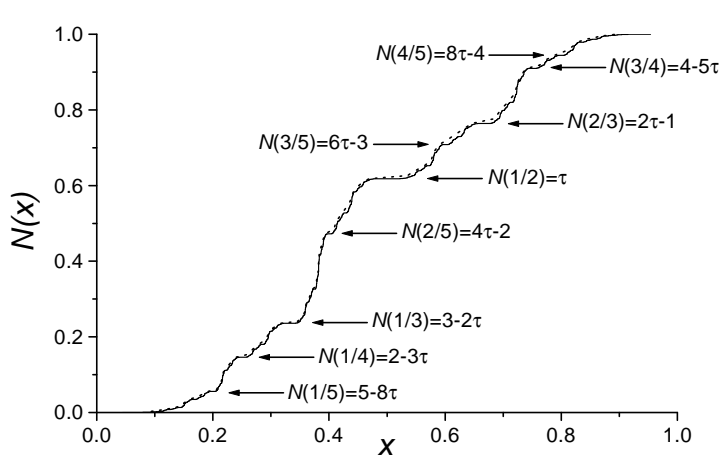

FIG. 2. The invariant measure $N(x)=\int_{0}^{x} d y f(y)$ obtained after 4 iterations (dotted line involving only $3^{4}=81$ different $x_{i}$ 's) and 10 iterations $\left(3^{10}=59049\right.$ different $x_{i}$ 's $)$.

The plateaux described by Eq. (47) are clearly visible in Fig. 2. The smaller the denominator $q$, the wider is the associated plateau as predicted by Eq. (47). In a pseudo-gap of $f$ (associated to a plateau for $N$ ), $N(p / q)=\left\{n_{p / q} \tau\right\}$, where $\{$.$\} denotes the positive frac-$ tional part and $n_{p / q}$ is a possibly negative integer. The values of $N(p / q)$ for a few small denominator fractions are presented in Fig. 2; they have been calculated by using Eqs. (63)-(66). Thus, a kind of "pseudo-gap labeling 
theorem" 25 applies here: for tight-binding Hamiltonians on $1 \mathrm{D}$ quasiperiodic chains associated to an irrational number $\alpha$ (see Ref. [25] for a general formulation), this theorem states that the integrated density of states (IDOS) displays plateaux (corresponding to the energy true gaps). In these plateaux, the IDOS takes values precisely of the form $\{n \alpha\}$. In one dimension, the IDOS is intimately related to the invariant measure $\nu[17]$. Therefore, it is not surprising that $\nu$ exhibits a similar behavior.

We now show how to calculate the Lyapunov exponent from the restricted invariant distribution $f(x)$. Take the basic formula, $\lambda=\int d r P(r) \ln r$, and perform the same massage as we did for the normalization condition in the beginning of this subsection. We obtain

$$
\begin{aligned}
& \lambda=\int_{0}^{1} d x f(x) g(x)+\mu, \\
& g(x)=\frac{1-\rho}{2} \sum_{k=0}^{\infty} \rho^{k} \ln \left(1-\left(\frac{1-x}{2 k+1}\right)^{2}\right), \\
& \mu=(1-\rho) \sum_{k=0}^{\infty} \rho^{k} \ln (2 k+1)=0.29320491137 \ldots
\end{aligned}
$$

We then numerically compute $\lambda$ via

$$
\lambda=\lim _{n \rightarrow \infty} \sum_{i=1}^{3^{n}} w\left(x_{i}\right) g\left(x_{i}\right)+\mu .
$$

For instance, starting from $x=1-\tau$, one reproduces 10 digits of $\lambda$ after only 8 iterations.

\section{GAUSSIAN RANDOM SEQUENCES}

Invariant measures exhibited by the Fibonacci random sequences appear fractal for all strengths of disorder. This is apparently caused by the discreteness of the disorder. Additionally, there is a transition between weak disorder, manifested by an invariant measure whose support is bounded, and strong disorder characterized by an invariant measure with unbounded support. This feature is seemingly caused by the boundness of the disorder. Hence, random sequences with unbounded continuous disorder distributions provide a natural counterpart to random sequences with binary disorder. As a specific example, we consider the Gaussian random recurrence,

$$
x_{n+1}=x_{n}+\beta z_{n} x_{n-1},
$$

where $z_{n}$ 's are independent identically distributed random variables with Gaussian probability density

$$
G(z)=\frac{1}{\sqrt{2 \pi}} e^{-z^{2} / 2}
$$

To analyze the Gaussian random recurrence, we again use the Ricatti variable $R_{n}=x_{n+1} / x_{n}$ which reduces Eq. (68) to the random map

$$
R_{n}=1+\frac{\beta z_{n}}{R_{n-1}}
$$

The invariant distribution $P(R)$ satisfies

$$
P(R)=\int d z G(z) \int d R^{\prime} P\left(R^{\prime}\right) \delta\left(R-1-\frac{\beta z}{R^{\prime}}\right),
$$

which can be re-written as

$$
P(R+1)=\frac{1}{\sqrt{2 \pi \beta^{2}}} \int d R^{\prime}\left|R^{\prime}\right| P\left(R^{\prime}\right) e^{-R^{\prime 2} R^{2} / 2 \beta^{2}} .
$$

Note two general features of the invariant distribution which directly follow from Eq. (72). One is a power-law large $R$ asymptotics,

$$
P(R) \rightarrow \sqrt{\frac{2}{\pi}} \frac{\beta P(0)}{R^{2}} \text { for } \quad R \gg \beta .
$$

Another unexpected feature is a weak logarithmic divergence at $R=1$ :

$$
P(R) \rightarrow \frac{2}{\pi} P(0) \ln \left(\frac{1}{|R-1|}\right) \quad \text { for } \quad|R-1| \ll \beta .
$$

Equation (74) follows from Eqs. (72)-(73).

We now turn to a perturbative analysis. While for the Gaussian random recurrence, there appears to be no threshold separating weak and strong disorder, different approaches should be implemented when $\beta \rightarrow 0$ and $\beta \rightarrow \infty$, respectively. In the former region, the regular perturbation theory applies while in the latter region one needs a singular perturbation theory.

\section{A. Weak disorder}

Equation $(70)$ shows that for weak disorder $(\beta \rightarrow 0)$, the magnitude of $R-1$ is comparable with $\beta$. This suggests to rescale the variable $R$ and the invariant distribution $P(R)$ according to

$$
R=1+\beta r, \quad P(R)=\beta^{-1} Q(r) .
$$

The normalization condition $\int d R P(R)=1$ now reads

$$
\int d r Q(r)=1
$$

The governing equation (72) becomes

$$
\frac{Q(r)}{G(r)}=\int_{-\infty}^{\infty} d r^{\prime}\left|1+\beta r^{\prime}\right| Q\left(r^{\prime}\right) e^{-r^{2}\left(\beta r^{\prime}+\beta^{2} r^{\prime 2} / 2\right)}
$$

In the small $\beta$ limit, we simplify Eq. (77) to

$$
\frac{Q(r)}{G(r)}=\int_{-\infty}^{\infty} d r^{\prime}\left(1+\beta r^{\prime}\right) Q\left(r^{\prime}\right) e^{-r^{2}\left(\beta r^{\prime}+\beta^{2} r^{\prime 2} / 2\right)} \text {. }
$$


The error caused by the above simplification is of order $Q(-1 / \beta)$. We will see that it vanishes as $\exp \left(-1 / 2 \beta^{2}\right)$ and therefore it can be ignored in perturbative analysis.

We are seeking a perturbative solution. The symmetry $\beta \leftrightarrow-\beta$ suggests an expansion in $\beta^{2}$ rather than $\beta$ :

$$
Q(r)=\sum_{n=0}^{\infty} \beta^{2 n} Q_{n}(r)
$$

We must also expand the exponent on the right-hand side of Eq. (77). This exponent is the generating function of the Hermite polynomials $H_{n}(r)$,

$$
\exp \left[-r^{2}\left(x+x^{2} / 2\right)\right]=\sum_{n=0}^{\infty} \frac{(-x r)^{n}}{n !} H_{n}(r)
$$

where $H_{n}(r)$ are defined via [23]

$$
H_{n}(r)=e^{r^{2} / 2}\left(-\frac{d}{d r}\right)^{n} e^{-r^{2} / 2}
$$

Thus, the Hermite polynomials make their appearance in the problem with non-Hermitian Hamiltonian.

Inserting Eqs. (79)-(80) into Eq. (78), we obtain

$$
\frac{Q_{n}(r)}{G(r)}=\sum_{k=1}^{n} \frac{r^{2 k-1}}{(2 k) !} H_{2 k+1}(r) \int d r^{\prime}\left(r^{\prime}\right)^{2 k} Q_{n-k}\left(r^{\prime}\right)
$$

In deriving Eq. 82, we also used the recursion relation for the Hermite polynomials [23]:

$$
H_{n+1}(r)=r H_{n}(r)-n H_{n-1}(r) .
$$

Solving Eq. (82) recursively yields

$$
\begin{aligned}
Q_{0}(r)= & G(r) \\
\frac{Q_{1}(r)}{G(r)}= & \frac{1}{2} r H_{3}(r) \\
\frac{Q_{2}(r)}{G(r)}= & \frac{3}{2} r H_{3}(r)+\frac{1}{8} r^{3} H_{5}(r), \\
\frac{Q_{3}(r)}{G(r)}= & 12 r H_{3}(r)+\frac{5}{4} r^{3} H_{5}(r)+\frac{1}{48} r^{5} H_{7}(r), \\
\frac{Q_{4}(r)}{G(r)}= & \frac{7857}{32} r H_{3}(r)+\frac{135}{8} r^{3} H_{5}(r) \\
& +\frac{7}{16} r^{5} H_{7}(r)+\frac{1}{384} r^{7} H_{9}(r), \\
\frac{Q_{5}(r)}{G(r)}= & \frac{1362843}{256} r H_{3}(r)+\frac{48675}{128} r^{3} H_{5}(r) \\
& +\frac{147}{16} r^{5} H_{7}(r)+\frac{3}{32} r^{7} H_{9}(r)+\frac{1}{3840} r^{9} H_{11}(r),
\end{aligned}
$$

etc. We will use these results to compute the Lyapunov exponent. The basic formula (8) now reads

$$
\lambda=\int_{-\infty}^{\infty} d r Q(r) \ln |1+\beta r|
$$

Expanding the logarithm and the invariant distribution, Eq. (79), and recalling that $Q(r)=Q(-r)$, we get

$$
\lambda=-\sum_{n=1}^{\infty} \beta^{2 n} \sum_{k=1}^{n} \frac{1}{k} \int_{0}^{\infty} d r r^{2 k} Q_{n-k}(r) .
$$

Inserting above expressions for $Q_{n}(n=0, \ldots, 5)$ into Eq. (85), we obtain the weak disorder expansion:

$$
\begin{aligned}
\lambda(\beta)= & -\frac{1}{2} \beta^{2}-\frac{9}{4} \beta^{4}-22 \beta^{6}-\frac{13197}{32} \beta^{8} \\
& -\frac{2374335}{256} \beta^{10}-\frac{118392093}{512} \beta^{12}+\mathcal{O}\left(\beta^{14}\right) .
\end{aligned}
$$

Interestingly, neither the $R^{-2}$ asymptotics (73) nor the logarithmic singularity (74) appear in the weak disorder expansion. Both these behaviors are non-perturbative. For instance, $P(0)$ which appears on the right-hand sides of Eqs. (73)-(74) scales as $\exp \left(-1 / 2 \beta^{2}\right)$, i.e., the behaviors Eqs. (73)-(74) are beyond the scope of perturbation techniques. Note also that the observation of the logarithmic singularity (74) requires probing a prohibitively tiny region $r \sim \exp \left[-\exp \left(-1 / 2 \beta^{2}\right)\right]$.

The occurrence of non-perturbative corrections (of order $\left.\exp \left(-1 / 2 \beta^{2}\right)\right)$ suggests that the radius of convergence of the series (86) is equal to zero. This is (non-rigorously) confirmed by the following approximate analysis of $\lambda(\beta)$. First, we write $R_{n} \approx 1+\beta z_{n}$, as the distribution of $R_{n-1}$ is strongly peaked at $R=1$ for small $\beta$. Within this approximation, and using the fact that the Gaussian distribution is an even function, we obtain

$$
\lambda(\beta) \approx \frac{1}{2} \int_{-\infty}^{\infty} d z G(z) \ln \left|1-\beta^{2} z^{2}\right| d z,
$$

which reproduces exactly the first term of Eq. (86). Now, expanding Eq. (87) in powers of $\beta^{2}$, is likely to lead to the correct qualitative behavior for the full general expansion. The generic term,

$$
-\frac{\beta^{2 n}}{2 n} \int_{-\infty}^{\infty} d z G(z) z^{2 n}=-\frac{\beta^{2 n} 2^{n-1}}{n \sqrt{\pi}} \Gamma\left(n+\frac{1}{2}\right)
$$

grows faster than any exponential, ensuring that the radius of convergence is indeed zero. Of course, we cannot exclude that for the actual expansion, subtle cancellations lead to a finite radius of convergence. However, the occurrence of non-perturbative corrections, and concrete ingredients of the argument presented above (mainly, the unboundness of the distribution $G(z)$ and the fact that the series $\ln (1+x)$ as a finite radius of convergence) which seem to persist in the general case, favor a zero radius of convergence and the asymptotic character of the series (86). This is very different from the case of random Fibonacci sequences where the weak disorder expansion has a finite radius of convergence and there were no trace of any non-perturbative contribution. 


\section{B. Strong disorder}

For $\beta \rightarrow \infty$, we again use the properly normalized Ricatti variable $y_{n}=x_{n+1} / x_{n} \sqrt{\beta}$. Equation (68) reduces to the random map

$$
y_{n}=\frac{z_{n}}{y_{n-1}}+\delta, \quad \delta \equiv \beta^{-1 / 2}
$$

The invariant distribution satisfies

$$
P(y+\delta)=\frac{1}{\sqrt{2 \pi}} \int_{-\infty}^{\infty} d \eta|\eta| P(\eta) \exp \left\{-\frac{y^{2} \eta^{2}}{2}\right\},
$$

and the Lyapunov exponent is given by Eq. (16) as in the Fibonacci case. Equation (89) suggests to seek a perturbative solution. In the zeroth order, one might set $\delta=0$ in Eq. (89). The corresponding invariant distribution $P_{0}(y)$ is an even function of $y$ which satisfies

$$
P_{0}(y)=\sqrt{\frac{2}{\pi}} \int_{0}^{\infty} d \eta \eta P_{0}(\eta) \exp \left\{-\frac{y^{2} \eta^{2}}{2}\right\} .
$$

Paradoxically, a (formal) solution to this equation,

$$
P_{0}(y)=\frac{A}{|y|}
$$

does not obey the normalization requirement. This indicates that the naive perturbation approach does not work and one must develop a singular perturbation theory. One still anticipates that $P_{0}(y)$ is given by Eq. (91) apart from the small and large scales, $|y| \sim \delta$ and $|y| \sim \delta^{-1}$, which are implied by the random map (88). Treating these scales as cutoffs allows us to normalize the solution (91) and to estimate the amplitude $A \approx(2 \ln \beta)^{-1}$. One can establish the existence of the cutoffs more rigorously. Using Eqs. (89), (91) one estimates $P(0) \sim A \delta^{-1}$ in agreement with the existence of the small scale cutoff $y \sim \delta$. The large scale cutoff already follows from Eq. (73) which now reads

$$
P(y) \rightarrow \sqrt{\frac{2}{\pi}} \frac{P(0)}{y^{2}} \quad \text { for } \quad y \gg \delta^{-1} .
$$

Note also that the deficiency of the naive perturbation approach is clear from the respective random map, $y_{n}=z_{n} / y_{n-1}$. Indeed, iterating the above map and taking the logarithm gives $\ln y_{n}=\sum(-1)^{n-k} \ln z_{k}$. The central limit theorem now asserts that the scale of the limiting the distribution grows indefinitely with $n$, namely $\ln y_{n} \sim \sqrt{n}$. Thus for $\delta=0$ already the basic assumption that $y_{n}$ approaches to a limiting distribution which does not depend on $n$ is incorrect.

We now present a computation of the zeroth order contribution to the Lyapunov exponent which does not require the knowledge of $P_{0}$. Denote by $\Lambda$ the zeroth order contribution to the Lyapunov exponent. We have

$$
\begin{aligned}
\Lambda & =2 \int_{0}^{\infty} d y P_{0}(y) \ln y \\
& =2 \sqrt{\frac{2}{\pi}} \int_{0}^{\infty} d \eta \eta P_{0}(\eta) \int_{0}^{\infty} d y \ln y \exp \left\{-\frac{y^{2} \eta^{2}}{2}\right\} \\
& =2 \sqrt{\frac{2}{\pi}} \int_{0}^{\infty} d \eta P_{0}(\eta) \int_{0}^{\infty} \frac{d t}{\sqrt{2 t}} e^{-t} \ln \left(\frac{\sqrt{2 t}}{\eta}\right) \\
& =\int_{0}^{\infty} d \eta P_{0}(\eta)\left[\Psi\left(\frac{1}{2}\right)+\ln 2-2 \ln \eta\right] \\
& =\frac{1}{2}\left[\Psi\left(\frac{1}{2}\right)+\ln 2\right]-\Lambda .
\end{aligned}
$$

In the second line we used Eq. (90); this step is not really rigorous though we think the final result is correct. The variable $t$ which appears in the third line has been defined via $t=y^{2} \eta^{2} / 2$; in the fourth line we used the digamma (psi) function, $\Psi(x)=\Gamma^{\prime}(x) / \Gamma(x)$ 23]; in the last line we used the normalization requirement and the definition of $\Lambda$. The above equation yields $\Lambda$ which can be simplified further by using the identity $\Psi(1 / 2)=-\gamma-2 \ln 2$, where $\gamma$ is the Euler constant. Finally,

$$
\Lambda=-\frac{\gamma+\ln 2}{4}=-0.317590711365 \ldots
$$

Our numerical results suggest that the strong disorder expansion involves powers of $(\ln \beta)^{-1}$ rather than $\beta^{-1}$ :

$$
\lambda(\beta)=\frac{1}{2} \ln \beta+\Lambda+\sum_{k=1}^{\infty} b_{k}(\ln \beta)^{-k} .
$$

Of course, it is hardly possible to probe higher order logarithmic terms numerically. However, plotting $\lambda-\frac{1}{2} \ln \beta$ versus $(\ln \beta)^{-1}$ gives a fairly straight line for $\beta>10^{4}$, with the slope $b_{1} \approx 0.557$, and a perfect fit to the above functional form, keeping a quadratic term in $(\ln \beta)^{-1}$, with $b_{2} \approx-0.52$ (see Fig. 3 ).

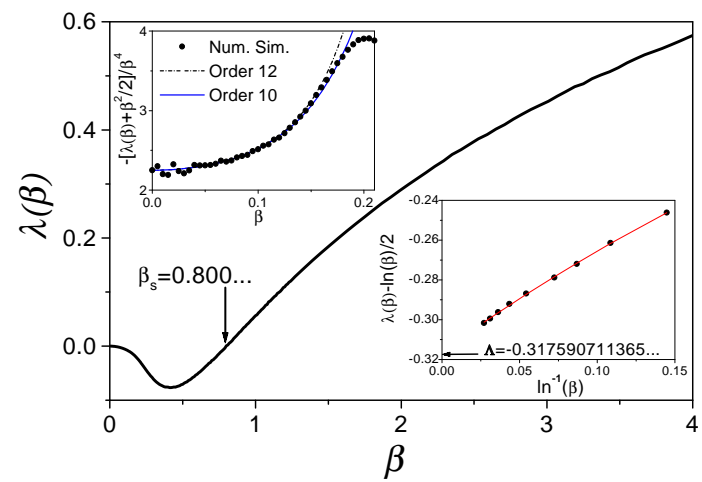

FIG. 3. We plot $\lambda(\beta)$ for Gaussian random sequences. The upper insert compares $-\left(\lambda(\beta)+\beta^{2} / 2\right) / \beta^{4}=9 / 4+22 \beta^{2}+\ldots$ obtained from the 10th and 12th order expansions in Eq. (86) to the result of numerical simulations. The lower insert is a quadratic fit in $(\ln \beta)^{-1}$ (see Eq. (94)) of $\lambda(\beta)-\frac{1}{2} \ln \beta$, for large $\beta$, leading to an extrapolated $\Lambda \approx-0.317$, with $b_{1} \approx 0.557$ and $b_{2} \approx-0.52$. 
Extrapolating the quadratic fit to $(\ln \beta)^{-1}=0$ yields $\Lambda \approx-0.317$, in good agreement with the theoretical prediction (94). Contrary to the Fibonacci case, the curve of $\lambda(\beta)$ appears perfectly smooth, and is certainly not fractal.

Finally, we note that asymptotic methods [23] should in principle allow to perform the strong disorder expansion more systematically.

\section{DISCUSSION}

The rich behavior exhibited by random Fibonacci numbers suggests avenues for further investigation. For instance, how to reconcile perturbative results in the large $\beta$ limit with non-perturbative results for $\beta=1$ ? This question is important as there appears to be just a single threshold $\beta=1 / 4$ and therefore $\beta=1$ lies within the large $\beta$ domain. This suggests qualitative similar behaviors which is not the case. The major difference between $\beta=1$ and $\beta \rightarrow \infty$ cases is manifested in extreme behaviors of the invariant measure. In the former case, it exhibits exponential asymptotics, Eqs. (44)-(45), while the latter is characterized by power-law asymptotics, $\nu(r) \sim r$ for $r \rightarrow 0$ and $\frac{1}{2}-\nu(r) \sim r^{-1}$ for $r \rightarrow \infty$. More generally, our perturbative results are infinitely smooth, in a gross disagreement with the behavior for $\beta=1$. Another (related) set of questions concerns the curve $\lambda(\beta)$ : Is it a fractal? Does it become genuinely smooth at least for sufficiently large $\beta$ ?

One could ask for a more complete characterization of the growth (decay) of the random Fibonacci numbers. A natural conjecture is $x_{n} \sim e^{\lambda n} n^{\omega}$. For the neutrally stable recurrence, $x_{n+1}=x_{n} \pm \beta_{s} x_{n-1}$ with $\beta_{s} \approx 0.70258$ chosen so that $\lambda\left(\beta_{s}\right)=0$, the above conjecture would imply the power-law behavior $x_{n} \sim n^{\omega_{s}}$.

Finally, it would be very interesting to analyze random Fibonacci numbers for the critical strength of disorder, $\beta=1 / 4$. This strength of disorder appears a bit more interesting than $\beta=1$ : It is hard to see what distinguishes $\beta=1$ from say $\beta=1.23456$, while the case of $\beta=1 / 4$ is certainly special as it separates the regions of weak and strong disorder.

We are grateful to H. Castillo, G. Oshanin, S. Redner, and P. Sharma for discussions. The work of PLK was supported by NSF Grant DMR9978902.
[1] J. H. Conway and R. K. Guy, The Book of Numbers (Copernicus, New York, 1996).

[2] http://www.ee.surrey.ac.uk/Personal/R.Knott/ gives a huge amount of of information on Fibonacci numbers. A mathematical periodical, the Fibonacci Quartely, is entirely devoted to the subject.

[3] R. V. Jean, Phyllotaxis: A Systematic Study of Plant Morphogenesis (Cambridge University Press, Cambridge, 1994).

[4] P. Bougerol and J. Lacroix, Products of Random Matrices with Applications to Schrödinger Operators (Birkhäuser, Boston, 1985).

[5] P. Diaconis and D. Freedman, SIAM Rev. 41, 45 (1999).

[6] H. Furstenberg, Trans. Amer. Math. Soc. 108, 377 (1963).

[7] D. Viswanath, Math. Comp. 69, 1131 (2000).

[8] M. Embree and L. N. Trefethen, Proc. R. Soc. Lond. 455, 2471 (1999).

[9] G. Oshanin, private communication.

[10] B. Halperin, Adv. Chem. Phys. 13, 123 (1967).

[11] B. Derrida and H. J. Hilhorst, J. Phys. A 16, 2641 (1983).

[12] F. J. Dyson, Phys. Rev. 92, 1331 (1953).

[13] H. Schmidt, Phys. Rev. 105, 425 (1957).

[14] M. Kappus and F. Wegner, Z. Phys. B 45, 15 (1981).

[15] B. Derrida and E. Gardner, J. Physique 45, 1283 (1984).

[16] A. Crisanti, G. Paladin, and A. Vulpiani, Products of Random Matrices in Statistical Physics (Springer, Berlin, 1992).

[17] J. M. Luck, Systèmes Désordonnés Unidimensionnels (Aléa-Saclay, 1992).

[18] L. P. Kadanoff and J. Swift, Phys. Rev. 165, 310 (1968); H. C. Fogedby, A. B. Eriksson, and L. V. Mikheev, Phys. Rev. Lett. 75, 1883 (1995); M. Bauer, D. Bernard, and J. M. Luck, J. Phys. A 34, 2659 (2001).

[19] N. Hatano and D. R. Nelson, Phys. Rev. B 56, 8651 (1997); D. R. Nelson and N. M. Shnerb, Phys. Rev. E 58, 1383 (1998); N. Hatano, Physica A 254, 317 (1998).

[20] I. Y. Goldsheid and B. A. Khoruzhenko, Phys. Rev. Lett. 80, 2897 (1998).

[21] J. Feinberg and A. Zee, Phys. Rev. E 59, 6433 (1999); Nucl. Phys. B 552, 599 (1999).

[22] B. Derrida, J. L. Jacobsen, and R. Zeitak, J. Stat. Phys. 98, 31 (2000).

[23] C. M. Bender and S. A. Orszag, Advanced Mathematical Methods for Scientists and Engineers (Springer-Verlag, New York, 1999).

[24] R. L. Graham, D. E. Knuth, and O. Patashnik, Concrete Mathematics, 2nd edition (Reading, Mass.: AddisonWesley, 1994).

[25] J. Bellissard, p. 99 in Statistical Mechanics and Field Theory, Mathematical Aspects, Lecture Notes in Physics, Vol. 257 (Springer-Verlag, Berlin, 1986). 